\title{
Synthesis and characterization of polybenzoxazole/graphene oxide composites via in situ polymerization
}

\author{
Jun Lim ${ }^{1}$, Min-Cheol Kim², Munju Goh ${ }^{1}$, Hyeounk Yeo ${ }^{1}$, Dong Geun Shin ${ }^{1}$, Bon-Cheol Ku ${ }^{1}$ and Nam-Ho You ${ }^{1, \star}$ \\ ${ }^{1}$ Carbon Convergence Materials Research Center, Institute of Advanced Composite Materials, Korea Institute of Science and Technology, \\ Wanju 565-905, Korea \\ ${ }^{2}$ Department of Chemistry, Chosun University, Gwangju 501-759 Korea
}

\section{Article Info}

Received 1 September 2013

Accepted 5 October 2013

*Corresponding Author

E-mail: polymer@kist.re.kr

Tel: $+82-63-219-8140$

\section{Open Access}

DOI: http://dx.doi.org/

10.5714/CL.2013.14.4.251

This is an Open Access article distributed under the terms of the Creative Commons Attribution Non-Commercial License (http://creativecommons.org/licenses/ by-nc/3.0/) which permits unrestricted non-commercial use, distribution, and reproduction in any medium, provided the original work is properly cited.

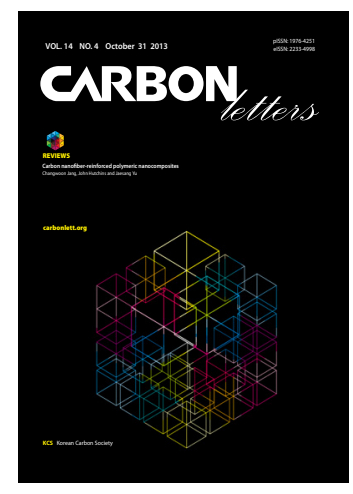

http://carbonlett.org

pISSN: $1976-4251$

elSSN: 2233-4998

Copyright $\odot$ Korean Carbon Society

\begin{abstract}
In this study, poly(amic acid) was prepared via a polycondensation reaction of 3,3'-dihydroxybenzidine and pyromellitic dianhydride in an N-methyl-2-pyrrolidone solution; reduced graphene oxide/polybenzoxazole (r-GO/PBO) composite films, which significantly increased the electrical conductivity, were successfully fabricated. GO was prepared from graphite using Brodie's method. The GO was used as nanofillers for the preparation of r-GO/PBO composites through an in situ polymerization. The addition of $50 \mathrm{wt} \% \mathrm{GO}$ led to a significant increase in the electrical conductivity of the composite films by more than sixteen orders of magnitude compared with that of pure PBO films as a result of the electrical percolation networks in the r-GO during the thermal treatment at various temperatures within the films.
\end{abstract}

Key words: fourier transform-infrared spectroscopy, polybenzoxazole, reduced graphene oxide, composite, electrical conductivity

\section{Introduction}

Polybenzoxazoles (PBOs) are rod-like polymers with good mechanical properties, superior thermal stability, good chemical resistance, low dielectric constant, and good processability [1,2]. Therefore, they have been widely used in IC chips, interlayer dielectrics for multilayer electronic devices, and high-end electronic packaging materials, as well as a variety of photoelectric devices [3]. The improved mechanical and optoelectronic properties have been demonstrated in carbon nanomaterials including carbon nanotubes (CNTs) and graphene into the polymer matrix [4,5].

Graphene, which is a two-dimensional carbonaceous material of $\mathrm{sp}^{2}$-bonded carbon atoms, has attracted significant attention due to its extraordinary electric, thermal property, and high surface area [6,7]. As with CNTs, graphenes have limitations in composite applications, because they are very difficult to disperse in both organic and inorganic solvents due to their significant van der Waals force [8]. In contrast, graphene oxide (GO) prepared via the chemical oxidation of graphite can meet this demand because it can be dispersed effectively in water and organic solvents due to its functional groups including epoxides, hydroxyls, ketones, and carbonyl groups [9-11]. Moreover, GO, which is obtained from graphite, is cost effective compared with graphene and CNTs.

Recently, many researchers have developed composite materials using GO and reducedGO (r-GO) as nanofillers for enhancing the mechanical and electrical properties using a small amount of GO $(<10 \mathrm{wt} \%)[12,13]$. However, few reports have focused on nanocomposites with a high $\mathrm{GO}$ content.

In this study, we report an effective method of preparing r-GO/PBO composite materials via in situ polymerization with a $\mathrm{GO}$ content of up to $50 \%$ for high performance nanocomposites. Using a high thermal conversion temperature above $550^{\circ} \mathrm{C}$ that results in a fully aromatic benzoxazole is an effective method of producing r-GO. The electrical and thermal properties of the r-GO/PBO composite 


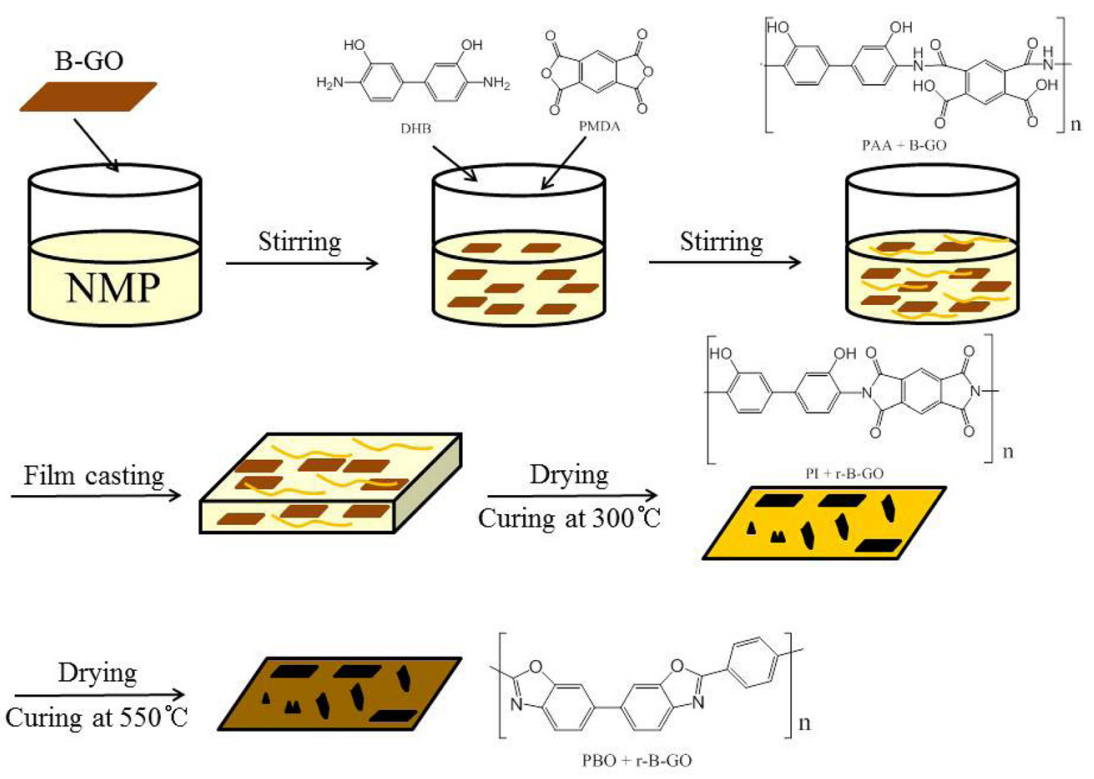

Scheme 1. Procedure for the preparation of r-GO/PBO composite films. r-GO: reduced graphene oxide, PBO: polybenzoxazole.

films were examined and scanning electron microscopy (SEM) was used to observe the morphology of the fractured surface of the films.

\section{Experimental}

\subsection{Synthesis of GO}

Graphite powders (99.9995\% purity) were purchased from Alfar Aesar (USA). Fuming nitric acid $\left(\mathrm{HNO}_{3}, 99.5 \%\right)$ and sodium chlorate $\left(\mathrm{NaClO}_{3}\right)$ were purchased from Sigma-Aldrich (Korea). Hydrochloric acid ( $\mathrm{HCl}, 35 \%)$ was obtained from Daejung Chemical (Korea). 3,3'-Dihydroxybenzidine (DHB) and pyromellitic dianhydride (PMDA) were obtained from TCI (Japan) and Lonza (China), respectively. N-methyl-2-pyrrolidone (NMP, 99.5\%) was also purchased from Sigma-Aldrich (Korea).

GO was synthesized using the Brodie method. First, $1 \mathrm{~g}$ of natural graphite and $8.5 \mathrm{~g}$ of $\mathrm{NaClO}_{3}$ were mixed using a stirrer at room temperature. Then, $9 \mathrm{~mL}$ of fuming nitric acid was slowly poured into the mixture using a dropping funnel in an ice bath and stirred for $24 \mathrm{~h}$ at ambient temperature. An additional 5 $\mathrm{mL}$ of fuming nitric acid was added into the mixture. Next, the mixture was heated at $60^{\circ} \mathrm{C}$ and stirred for $24 \mathrm{~h}$. After that, the mixture was neutralized using $200 \mathrm{~mL}$ of deionized (DI) water and washed with $100 \mathrm{~mL}$ of $3 \mathrm{M} \mathrm{HCl}$. After filtration, the neutralized mixture was dried in a vacuum oven at $50^{\circ} \mathrm{C}$ for $24 \mathrm{~h}$.

\subsection{Synthesis of poly(amic acid) and its nano- composite solutions}

PMDA (0.500 g, $2.3 \mathrm{mmol})$ was added to a solution of DHB $(0.496 \mathrm{~g}, 2.3 \mathrm{~mm})$ in NMP $(10 \mathrm{~mL})$. An additional NMP solution was added until the solid content was adjusted to $15 \%$ by weight. The mixture was stirred at room temperature for $24 \mathrm{~h}$ in order to produce a viscous and homogeneous poly(amic acid) (PAA). Using the $1 \mathrm{wt} \% \mathrm{GO} / \mathrm{PAA}$ composite solution as an example, the detailed synthesis procedures were as follows: $0.010 \mathrm{~g}$ of B-GO was added to the NMP solution and the mixture was subjected to sonication and homogenization for $1 \mathrm{~h}$. Then, $0.496 \mathrm{~g}$ of DHB was added to the GO solution. Next, $0.500 \mathrm{~g}$ of PMDA was added to the mixture. Finally, $1 \mathrm{wt} \% \mathrm{GO} / \mathrm{PAA}$ solution was obtained with the desired viscosity. A series of GO/PAA composite solutions with different GO loadings (1 50 wt\%) were prepared using the same method.

\subsection{Preparation of pure polyimide and its composite films}

The conventional solvent casting method was used to fabricate the PAA and GO/PAA composite films. A horizontal bar was used to control the thicknesses of the films with a casting speed of $6 \mathrm{~mm}$. Pure PAA and GO/PAA solutions with a thickness of approximately $750 \mu \mathrm{m}$ were cast onto the commercial polyimide (PI) film. All cast films were dried at $90^{\circ} \mathrm{C}$ for $2 \mathrm{~h}$ in a vacuum oven to remove the residual solvent. All films were heated under a nitrogen atmosphere for $30 \mathrm{~min}$ at $100^{\circ} \mathrm{C}, 150^{\circ} \mathrm{C}$, $200^{\circ} \mathrm{C}, 300^{\circ} \mathrm{C}, 350^{\circ} \mathrm{C}, 450^{\circ} \mathrm{C}$, and $550^{\circ} \mathrm{C}$.

\subsection{Characterizations}

The chemical reaction was confirmed using a Fourier transform-infrared spectroscopy (FT-IR Spectrophotometer, Nicolet IS10, USA). The electrical conductivities were measured using a four-probe resistivity meter (FPP-RS8, Dasol Engineering, Korea) and a super megohmmeter (SM-8200, Hioki, Japan). The thermogravimetric analysis (TGA) was performed in order to investigate the stability and thermal conversion from PAA to $\mathrm{PBO}$ in a nitrogen atmosphere at a temperature increase of $10^{\circ} \mathrm{C} / \mathrm{min}$ using a TGA Q50 (TA Instruments, USA). The analyses of the fractured cross-sections were observed using a field emission SEM (FE-SEM, FEI, USA). 


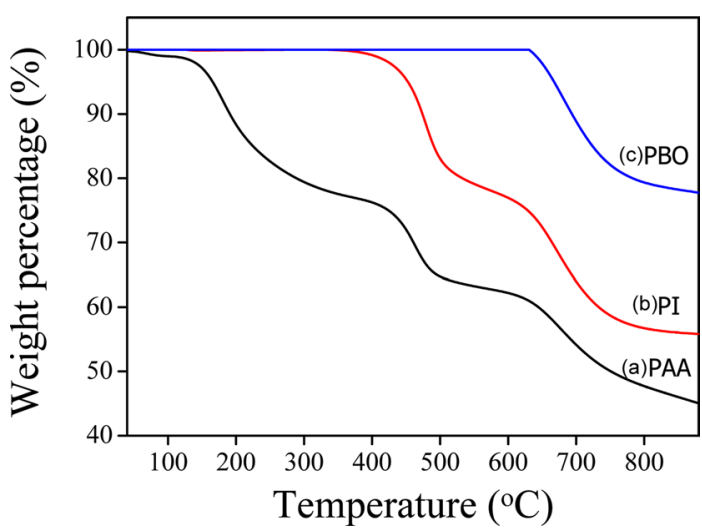

Fig. 1. Thermogravimetric analysis thermograms of (a) PAA, (b) $\mathrm{Pl}$, and (c) PBO. PAA: poly(amic acid), PI: polyimide, PBO: polybenzoxazole.

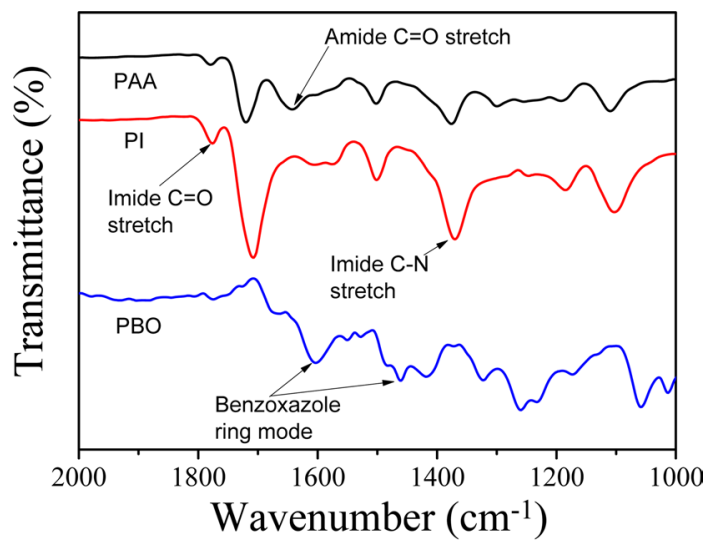

(a)

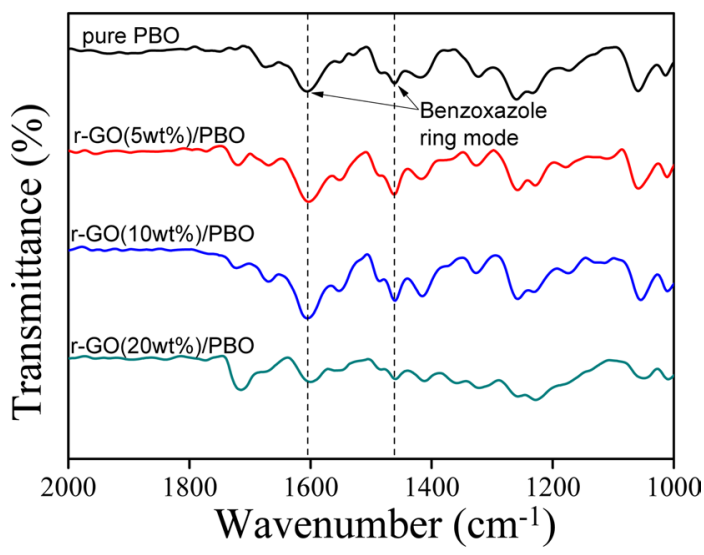

(b)

Fig. 2. Fourier transform-infrared spectroscopy spectra of the (a) $\mathrm{PAA}, \mathrm{PI}, \mathrm{PBO}$ and (b) r-GO/PBO composite films. PAA: poly(amic acid), PI: polyimide, PBO: polybenzoxazole, $r-G O$ : reduced grephene oxide.

\section{Results and Discussion}

$\mathrm{GO}$ as the filler in the synthesis of PAA was obtained using the Brodie method and then dispersed in the NMP solution using ultrasonication for a uniform dispersion. The GO/PAA films
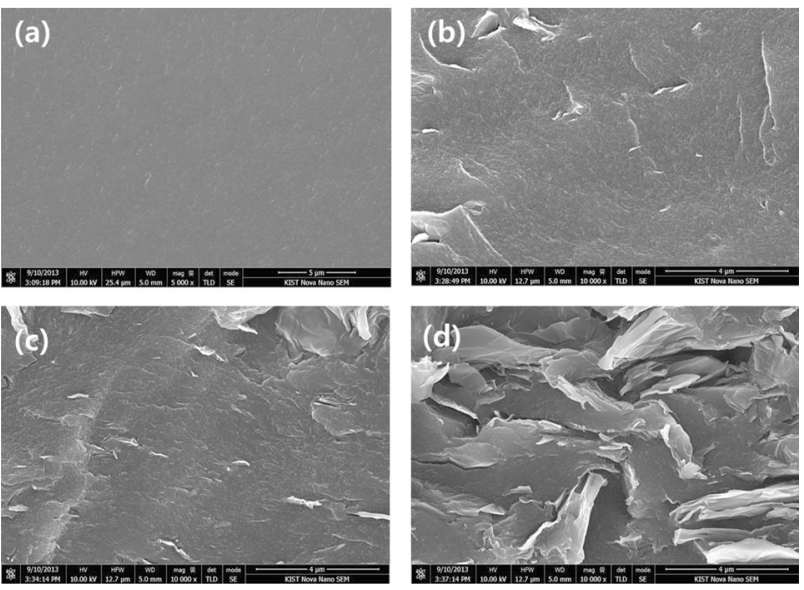

Fig. 3. Scanning electron microscopy micrographs for the fractured surfaces of (a) pure PBO, (b) r-GO/PBO composite containing $5 \mathrm{wt} \% \mathrm{GO}$, (c) r-GO/ $\mathrm{PBO}$ composite containing $10 \mathrm{wt} \% \mathrm{GO}$, and (d) r-GO/PBO composite containing 20 wt $\%$ GO. PBO: polybenzoxazole, r-GO: reduced grephene oxide.

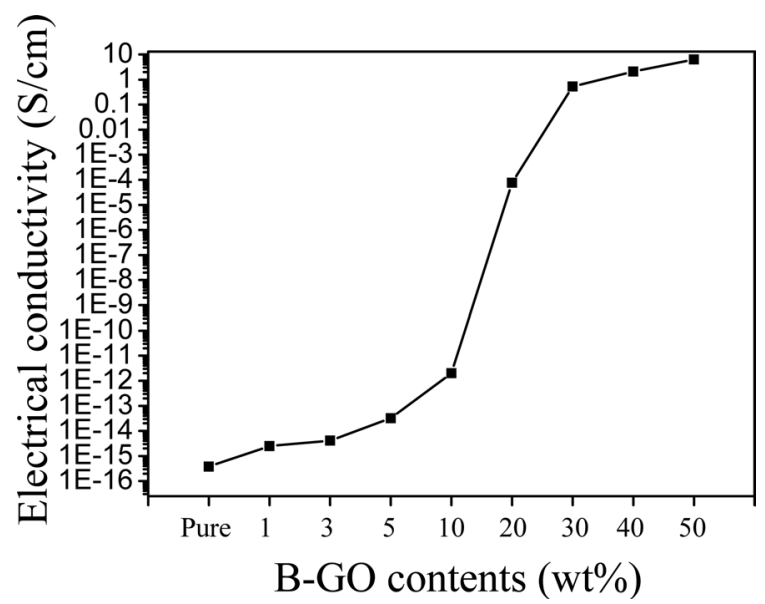

Fig. 4. Electrical conductivity of pure $\mathrm{PBO}$ and its composites. $\mathrm{PBO}$ : polybenzoxazole, GO: graphene oxide.

were prepared via casting onto the PI film using heat treatment in order to obtain the r-GO/PBO composite films as shown in Scheme 1. Fig. 1 presents the TGA thermograms of PAA before and after the thermal treatment. The first weight loss resulted from the thermal dehydration from PAA to PI.

The second weight loss originating from the cyclocarboxylation $\left(-\mathrm{CO}_{2}\right)$ was attributed to the thermal conversion from PI to PBO and appeared between $400-500^{\circ} \mathrm{C}$. The FT-IR spectra of PAA, PI, PBO, and their composites are shown in Fig. 2. Prior to heating, the characteristic absorption peaks that originated from the $\mathrm{C}-\mathrm{NH}$ stretching (amide II) and $\mathrm{C}=\mathrm{O}$ in $\mathrm{CONH}$ (amide I) stretching bands were identified at 1649 and $1538 \mathrm{~cm}^{-1}$. After the thermal conversion, the absorptions peaks originating from the imide moiety at $1716 \mathrm{~cm}^{-1}(\mathrm{C}=\mathrm{O}$ symmetric stretching), 1370 $\mathrm{cm}^{-1}$ (C-N stretching), and $723 \mathrm{~cm}^{-1}(\mathrm{C}=\mathrm{O}$ bending of imide) were observed. Finally, the absorption peaks at 1600 and 1460 $\mathrm{cm}^{-1}$ were confirmed to form the benzoxazole ring [14]. The FTIR spectra of the r-GO/PBO composites at 1600 and $1460 \mathrm{~cm}^{-1}$ that result from the PBO unit were observed as shown in Fig. 2b. However, there was no significant difference between PBO and 
its composites. These results indicate that the $\mathrm{r}-\mathrm{GO} / \mathrm{PBO}$ composite films were successfully obtained. The fractured surfaces of the pure $\mathrm{PBO}$ and $\mathrm{r}-\mathrm{GO} / \mathrm{PBO}$ composite films were observed via SEM as shown in Fig. 3. As shown in Fig. 3a, the fractured surfaces of the pure PBO film were relatively smooth compared with the r-GO/PI composite containing 5 to $20 \mathrm{wt} \%$ of GO in Figs. 3b-d.

The electrical conductivities of the pure $\mathrm{PBO}$ and $\mathrm{r}-\mathrm{GO} / \mathrm{PBO}$ composite films were measured using the four-point probe unit. As shown in Fig. 4, the electrical conductivity of each sample was increased as the GO loading increased. The electrical conductivity of the pure PBO was $3.83 \times 10^{-16} \mathrm{~S} / \mathrm{cm}$. However, the electrical conductivity of the $\mathrm{r}-\mathrm{GO} / \mathrm{PBO}$ composite containing $50 \mathrm{wt} \% \mathrm{GO}$ was $6.31 \mathrm{~S} / \mathrm{cm}$, which is an increase of more than sixteen orders of magnitude more than that of pure PBO film. This occurred because the $\mathrm{r}-\mathrm{GO}$ induced electrical percolation networks in the composites.

\section{Conclusions}

Using the Brodie method, GO was prepared from graphite as nanofillers for the preparation of $\mathrm{r}-\mathrm{GO} / \mathrm{PI}$ composites. The $\mathrm{PBO}$ was prepared via a polycondensation reaction followed by thermal curing at an elevated temperature and $\mathrm{r}-\mathrm{GO} / \mathrm{PBO}$ composite films, which significantly increased the electrical conductivity, were successfully fabricated. A FT-IR was performed in order to confirm the thermal conversion from PI to PBO and its composites with GO. The electrical conductivity of the r-GO/ PBO composite film containing $50 \mathrm{wt} \%$ of GO was $6.31 \mathrm{~S} / \mathrm{cm}$, which is sixteen orders of magnitude more than that of pure PBO film due to the thermal conversion temperature above $550^{\circ} \mathrm{C}$ used to produce $\mathrm{PBO}$

\section{Acknowledgements}

This work was supported by two grants from the Korea Institute of Science and Technology (KIST) Institutional program (Nos. 2Z03870, 2Z03800). It was also supported by a grant from the Ministry of Trade, Industry \& Energy of Korea (no. 2MR0760).

\section{References}

[1] Yokota R, Horiuchi R, Kochi M, Soma H, Mita I. High strength and high modulus aromatic polyimide/polyimide molecular composite films. J Polym Sci C, 26, 215 (1988). http://dx.doi.org/10.1002/ pol.1988.140260501.

[2] Ueda M, Nakayama T. A new negative-type photosensitive polyimide based on poly(hydroxyimide), a cross-linker, and a photoacid generator. Macromolecules, 29, 6427 (1996). http:// dx.doi.org/10.1021/ma9605560.

[3] Tokoh A. Photosensitive Polymers, Kansai Research Institute, Japan, 84 (1999).

[4] Dai L, Mau AWH. Controlled synthesis and modification of carbon nanotubes and $\mathrm{C}_{60}$ : carbon nanostructures for advanced polymeric composite materials. Adv Mater, 13, 899 (2001). http:// dx.doi.org/10.1002/1521-4095(200107)13:12/13<899::AIDADMA899>3.0.CO;2-G

[5] Kitagawa T, Ishitobi M, Yabuki K. An analysis of deformation process on poly- $p$-phenylenebenzobisoxazole fiber and a structural study of the new high-modulus type PBO HM+ fiber. J Polym Sci B, 38, 1605 (2000). http://dx.doi.org/10.1002/(SICI)10990488(20000615)38:12<1605::AID-POLB50>3.0.CO;2-Z.

[6] Novoselov KS, Geim AK, Morozov SV, Jiang D, Katsnelson MI, Grigorieva IV, Dubonos SV, Firsov AA. Two-dimensional gas of massless Dirac fermions in graphene. Nature, 438, 197 (2005). http://dx.doi.org/10.1038/nature04233.

[7] Castro Neto AH, Guinea F, Peres NMR, Novoselov KS, Geim AK The electronic properties of graphene. Rev Mod Phys, 81, 109 (2009). http://dx.doi.org/10.1103/RevModPhys.81.109.

[8] Lee S, Kim YJ, Kim DH, Ku BC, Joh HI. Synthesis and properties of thermally reduced graphene oxide/polyacrylonitrile composites. J Phys Chem Solids, 73, 741 (2012). http://dx.doi.org/10.1016/j. jpcs.2012.01.015.

[9] Compton OC, Nguyen ST. Graphene oxide, highly reduced graphene oxide, and graphene: versatile building blocks for carbonbased materials. Small, 6, 711 (2010). http://dx.doi.org/10.1002/ smll.200901934.

[10] Cai W, Piner RD, Stadermann FJ, Park S, Shaibat MA, Ishii Y, Yang D, Velamakanni A, An SJ, Stoller M, An J, Chen D, Ruoff RS. Synthesis and solid-state NMR structural characterization of ${ }^{13} \mathrm{C}$-labeled graphite oxide. Science, 321, 1815 (2008). http:// dx.doi.org/10.1126/science.1162369.

[11] Lerf A, He H, Forster M, Klinowski J. Structure of graphite oxide revisited. J Phys Chem B, 102, 4477 (1998). http://dx.doi. org/10.1021/jp9731821.

[12] Luong ND, Hippi U, Korhonen JT, Soininen AJ, Ruokolainen J, Johansson LS, Nam JD, Sinh LH, Seppälä J. Enhanced mechanical and electrical properties of polyimide film by graphene sheets via in situ polymerization. Polymer, 52, 5237 (2011). http://dx.doi. org/10.1016/j.polymer.2011.09.033.

[13] Park OK, Hwang JY, Goh M, Lee JH, Ku BC, You NH. Mechanically strong and multifunctional polyimide nanocomposites using amimophenyl functionalized graphene nanosheets. Macromolecules, 46, 3505 (2013). http://dx.doi.org/10.1021/ma400185j.

[14] Chang JH, Park KM, Lee SM, Oh JB. Two-step thermal conversion from poly(amic acid) to polybenzoxazole via polyimide: Their thermal and mechanical properties. J Polym Sci B, 38, 2537 (2000). http://dx.doi.org/10.1002/1099-0488(20001001)38:19<2537 $\because$ AID-POLB50 $>3.0 . \mathrm{CO} ; 2-\mathrm{V}$ 